\title{
EDICIÓN, COMUNIDAD, CIUDADANÍA: CONSIDERACIONES DE UNA EDIAUTORA EN PROVINCIAS DE MÉXICO Y CHILE
}

\author{
Carmen Violeta Avendaño ${ }^{1}$
}

\section{Resumen/Abstract}

Se dice que las editoriales independientes son las que sostienen la biblio diversidad. Creo que es esa misma responsabilidad la que convierte la edición independiente en una forma de resistencia cívico-comunitaria. Pero la biblio diversidad que custodiamos es solo la punta del iceberg de la diversidad sofocada por un colonialismo en erosión. En esa comunidad millonaria de lectoras y lectores que aprendieron la lengua del amo hay mucha escritura por representar.

Palabras clave: edición independiente, comunidad, público, bibliodiversidad, autoedición

EDITION, COMMUNITY, CITIZENSHIP: CONSIDERATIONS OF A PUBLISHER IN THE PROVINCES OF MEXICO AND CHILE

It is said that bibliodiversity relies in independent editors. I believe it is that responsability what makes independent edition a form of civic-community resistance. But that bibliodiversity we guard is just the tip of the iceberg of a diversity that has been suffocated by an eroding colonialism. In that millionary community of readers that learnt the master's tongue, there is plenty of writing waiting for representation.

Keywords: independent edition, community, public, bibliodiversity, desktop publishing

Un libro, mientras no se lee, es solamente un ser en potencia, tan en potencia como una bomba que no ha estallado. Y todo libro ha de tener algo de bomba, de acontecimiento que al suceder amenaza y pone en evidencia, aunque sólo sea con su temblor, a la falsedad.

María Zambrano

\footnotetext{
${ }^{1}$ Chilena. E-mail: edicionesmoneda@gmail.com
} 


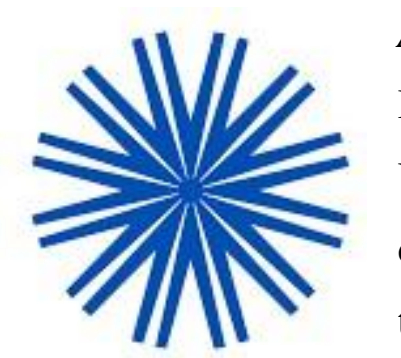

\section{Asuntos mundanos}

\section{I}

Un libro embodegado es un libro reproducido, no publicado. Un libro desconocido para los libreros, escondido en las repisas, tampoco se encuentra del todo publicado. 300 ejemplares no son 300 lectores. Quienes seguimos empecinadas en este no negocio sabemos que el lanzamiento es solo el inicio de un largo y tortuoso camino a las vitrinas, que nos puede llevar años, aún en aquellas librerías del barrio, pueblo o ciudad que habitamos. Los libros están todos pidiendo la palabra al mismo tiempo, pero algunos extienden la mano con billetes. Los más pedidos son los más exhibidos. Los más exhibidos son los más leídos. Entre ellos la política, la biografía, la novela, la autoayuda, hablan más fuerte, muchas veces, que la poesía, el cuento, el ensayo. Esto hace que la renovación sea lenta, por más que todos los meses haya "novedades" en tanto esas vienen a reemplazar novedades muy similares.

\section{II}

El espacio en vitrina, saturado por una aparente abundancia donde prima el libro-mercancía, es inaccesible a las editoriales independientes sin distribución, dado el costo administrativo que le genera a las librerías la multiplicación de los proveedores y el poco rédito que le significa exhibir publicaciones sin aparato publicitario ni criterios complacientes. La prioridad a lo que vende también opera con las distribuidoras al momento de integrar una nueva editorial a su cartera, así como la amplitud de su catálogo. Pero aún si una editorial independiente logra subsistir el tiempo necesario como para ofertar un catálogo amplio y conseguir ser aceptada en una distribuidora, pagará un costo elevado a la hora de los porcentajes en la consignación. El retorno es prácticamente inviable, para volver a editar y aún más generar ganancia por derechos de autor, desalentando a las nuevas voces. Estos factores que obstaculizan la permanencia de las editoriales independientes afectan más a las dirigidas por mujeres, en la medida en que sus redes laborales son más frágiles; y más todavía a las que trabajan fuera de la capital. En este sentido ser ediautora es tirar guijarros contra las altas vitrinas de la indiferencia.

\section{III}

El precio del libro refleja la cadena del libro y la jerarquía de cada eslabón. El precio justo se relaciona con la comprensión de los costos materiales y creativos de producción, con la cadena de oficios que sostienen al libro: escribir, diseñar, editar, traducir, imprimir, etc. La imprenta y la librería son los dos 
eslabones más costosos al momento de calcular el precio: una porque detenta el poder industrial de la multiplicación (a mayor cantidad menos precio por unidad) y la otra con un $40 \%$ de comisión justificado en apariencia por el costo de un lugar en la ciudad, y amparado en el bajo ritmo de ventas. Entremedio los eslabones creativos: escritura, diseño, edición, son los más castigados.

\section{IV}

El precio del libro afecta tanto a la producción como a la circulación. El precio fijo obedece a la comprensión del escenario actual donde conviven distintas formas de venta (la web de la editorial, mercado libre, Amazon, una librería de barrio o de Instagram; una feria). Para salir de una cultura lectora minoritaria y elitista se requiere la mayor cantidad de puntos de venta posibles, concebidos como puntos de encuentro con el lector, donde el precio fijo elimina problemas de competencia que podrían desincentivar la exposición de un título, y hace que los diversos actores de la venta de libros funcionen como un frente común, sin dejar escapar ninguna oportunidad de enlazar con los lectores posibles. La estrategia del descuento y el remate, pan para hoy y hambre para mañana, permea tanto en la industria masiva como en la más artesanal de las editoriales.

\section{V}

Entre el libro comercial y el artesanal está uno que podemos llamar neorepublicano, producido industrialmente pero fuera de un sistema de acumulación de riqueza: la diferencia de ventas entre el libro artesanal y el neorepublicano es menor que la diferencia de ventas este último y el comercial. Mi reticencia a adentrarme en el modo artesanal va más allá de la torpeza de mis manos; viene de mi experiencia como autora de una preciosa editorial michoacana, con libros de tapa dura que habitualmente imprime 50 ejemplares; para mi libro Madre Sol se hicieron 100 y aunque me consta que sigue circulando, es prácticamente un mito. A lo que aspiro, ingenuamente, con tirajes industriales mínimos, es a una utopía, y no me refiero a la de cubrir los gastos o a vender poesía, sino a cubrir una cantidad republicana de lectores.

\section{VI}

Si las independientes vemos a las comerciales como editoriales sin alma, ellas nos miran a nosotras como empresas sin capital. Suponemos cómo se hace un libro sin alma: se examina su contenido en función de cuánta ganancia puede producir. Pero ¿cómo se hace un producto sin capital? Y más aún, ¿por qué? Son 
los milagrosos fondos estatales del libro los que sujetan en gran medida la producción "independiente" a su reñida convocatoria anual, haciendo que las novedades "independientes" aparezcan a un ritmo demasiado lento en comparación a las comerciales. La edición fondeada podríamos llamarle, atendiendo al hecho de que una vez impreso el libro permanece en cajas un tiempo considerable antes de circular más ampliamente.

\section{La edición como ejercicio de ciudadanía}

I

Si pensamos en el libro artesanal como una respuesta comunitaria, es decir un libro que corresponde en su dinámica a un ámbito específico ¿Cuántas copias se requieren para que un libro industrial se considere una respuesta republicana? Si un libro fuera una localidad, ¿sería una toma, un sector, un barrio, un pueblo, una ciudad, un área metropolitana, un pequeño o gran país, un continente? El libro es un espacio para llevar. Un lugar en tránsito y de tránsito, destinado a atravesar y ser atravesado por la diversidad de configuraciones territoriales a lo largo del tiempo.

El dilema de la edición independiente estriba en las dificultades para que ese traslado se efectúe en un mundo estructurado para el tráfico de bienes comerciales a gran escala. El submundo de la gente que lee contiene un submundo de gente que lee literatura y otro mucho más pequeño de la gente que lee literatura editada con independencia de su rédito. Por otra parte, operar con los libros como otra mercancía más, en función de oferta y demanda es renunciar al ejercicio de un criterio compartido libremente. Ante la dificultad para circular por las autopistas modernas, se apuesta a sostenerse en el tiempo, se apuesta a la transformación social.

\section{II}

Una historia ejemplar de la circulación del libro de la capital a la provincia y de regreso es la de Antonio Porchia, anarquista argentino "de barco", nacido en Italia y renacido en La Boca, con su Voces de 1943, costeadas por él, a instancias de sus amigos anarquistas de la agrupación "Impulso". Los paquetes de Voces estorbaban la pasada, hasta que las donó a la Sociedad Protectora de Bibliotecas Populares. Y uno de los lectores en provincia de estas bibliotecas envió un comentario a la revista Sur dirigida por Victoria Ocampo, que cae en manos de Roger Caillois. Fascinado por las Voces las tradujo al francés y se 
publicaron en Francia. Desde Francia llamaron la atención en Argentina, y entonces llegaron a las vitrinas bonaerenses, donde una vez entró Porchia sin el dinero suficiente para comprarlas.

\section{III}

El problema del alcance radica menos en la cantidad de ejemplares que en los circuitos que abarca el efecto de ondas de los contenidos. Es decir que ese libro sí, es público, pero público entre quiénes. Dónde. El ámbito restringido de circulación llama la atención en un país donde el $96 \%$ de la población técnicamente puede leer y escribir.

¿Por qué los libros tienen baja demanda, si vivimos en la era de la alfabetización mundial, con múltiples recursos para la impresión y el envío? Tal vez porque este mundo de oportunidades para la lectura se ha levantado desde un sistema educativo colonial, patriarcal, capitalista. De hecho, la verdadera escala republicana del tiraje hoy es el de aquellos libros destinados a la escuela pública. En la base de este sistema está una doble imposición: la lengua nacional, por encima de cualquier otra realidad del sentido; y la escritura, con todo el aparato legal, epistémico y comercial, por encima de la vida oral. ¿Quién va a desear la lectura así? ¿cómo podía ser la lectura en semejante marco escolar sino ese martirio de ilustraciones inhóspitas, esa carrera por devorar capítulos, como hamburguesas? ¿Cómo iba a ser sino una institución para desalentar la lectura y ensalzarla al mismo tiempo?

\section{IV}

Gran parte de la literatura que reverenciamos está escrita en 6 de las 6000 lenguas que existen, de las cuales $43 \%$ se encuentran en peligro de extinción, básicamente a causa de la aplanadora educativa monolingüe de los sistemas coloniales. Solo unos pocos centenares de idiomas han tenido el privilegio de incorporarse a los sistemas educativos y al dominio público, y menos de cien se emplean en el mundo digital. Cuán a favor de este continente sería tener el castellano como lengua franca si fuera opcional o qué deseada la posibilidad de escribir tu propia lengua si no estuviera vetada de las matemáticas, la historia, el cajero, la tienda, el hospital, el museo, la comisaría.

\section{$\mathrm{V}$}

Visitando una radio comunitaria indígena en San Juan Parangaricutiru, junto al volcán Paricutín, estuve por primera vez en un espacio público hablado en una lengua indígena, donde la tecnología de la comunicación le permitió al pueblo solucionar un problema de contaminación auditiva (los altavoces 
saturaban este pueblo de 16.000 habitantes) y otorgar un lugar de dignidad a la lengua p'urhépecha, que el sistema escrito escolar, le había negado. ¿Espacio público o comunitario? ¿Integrado no por ciudadanos anónimos o por miembros de la comunidad identificados en tanto tales?

\section{VI}

La escuela que conocemos hace de la lectura una actividad obligatoria saturada de libros despersonalizados, llamados "de texto", que no permiten la libre circulación por los contenidos autónomos. Una educación abierta a la cultura oral y al tránsito libre por los contenidos, una educación para disfrutar la vida y no para padecerla como ratón de laboratorio de mercado laboral, será la que fomente la lectura.

\section{Selfie de una ediautora}

\section{I}

Reconocer la edición como ejercicio de ciudadanía implica tomar una postura frente al abuso de la cultura letrada en los procesos coloniales. Reconocerme mestiza significa ser hija de una tradición escrita y de una tradición oral. Dentro de mi ascendente escrito vengo del aristocrático 12\% del mundo que en 1820 era letrado. Un 12\%, con participación mínima de mujeres y demás otredades. Varios de los libros considerados clásicos fueron escritos antes de esta fecha, lo que indica una enorme diversidad que solo recientemente está empezando a acceder a un lugar autoral en la literatura donde trabajaba de personaje, musa o paisaje.

\section{II}

Reconocer mi ascendente oral no pasa solo por tener antecesores hablantes de una lengua predominantemente oral - que en el fondo son todas - sino también reconocerme como mujer, es decir como sujeto que se ha desempeñado históricamente en el ámbito desprestigiado del conocimiento oral. A un mismo tiempo implica reconocer formas mezcladas con la tecnología y la cultura popular, y los terrenos móviles, como los muros, las pancartas, las redes, en dirección contraria al libro en tanto portador de prestigio.

III 
La alfabetización en lengua dominante determina que la materia que en Chile se denomina "lenguaje" y en México "español”, es inconcebible fuera de la cultura escrita, y la cultura escrita es opresivamente castellana, aunque con una sumisión ambigua al inglés, la lengua de los negocios, el espectáculo, la música; y residualmente al francés, el alemán, el italiano, como lenguas de lo culto, imbricadas con la tradición literaria occidental. Negociar con esa tradición es parte del trabajo editorial.

\section{IV}

¿De qué manera me ubico, en lo concreto de mi proyecto ediautoral? En Ediciones Moneda asumo mi parte de esa herencia desde la apropiación, con Stéphane Mallarmé (Francia, 1842-1898) y Miltos Sajturis (Grecia, 1919-2005), que representa la traducción por hablantes de Chile y en particular traductoras. Mallarmé ha sido una influencia indirecta en América Latina (a través de Vallejo, Huidobro, Lezama), por falta de buenas traducciones íntegras bien difundidas. Frente al Muro, de Miltos Sajturis, es el primer libro unitario del poeta griego que se traduce al castellano. Ambas obras cubren huecos significativos dejados por un eurocentrismo disparejo. Por otra parte, el libro que antologo y prologo de Álvaro Ruiz (Ottawa, 1953) Horizonte Vertical, responde tanto al apagón cultural de dictadura, que afectó a su generación en la publicación y difusión de obra, como a la misma edición independiente de tiraje acotado en la que Ruiz ha circulado sin llegar a darse a conocer a un público más amplio. Actualmente proyecto tres publicaciones, una de ellas relacionadas con la oralidad - el testimonio- otra con la biografía de una mujer clave en la historia de América Latina y otra con la obra más reciente de Margaret Randall (Nueva York, 1936), poeta publicada en la mayoría de los países de centro y sur de América, pero aún no en Chile. En cuanto a mi autoedición, (Nada significa nada 2019), se trata de un diálogo con las formas orales.

$\mathrm{V}$

¿Por qué me hice editora? Es posible que algo haya tenido que ver mi madre, su experiencia en las publicaciones periódicas El Rebelde y Miradas en Chile, y El Séptimo Sueño, en México; su trabajo como diseñadora gráfica, en un tiempo de maquetas con cartulina y papel albanene. Me tocó verla componer portadas, hacer de modelo, acompañarla a Franklin en busca de objetos para fotografiar, a la imprenta a ver las pruebas, pelear por un color, pararlo todo por una errata. A mi padre, como impresor, se le atribuye una única obra: entradas falsificadas para el concierto de Silvio Rodríguez. 
Desde un inicio fui autoeditora. Cuando hice mi primer cómic a los 14 años sentí la necesidad de crear para él una entidad, un desde dónde, una identidad pictográfica, simbólica: un botón. Y cuando escribí mi primer libro, darle yo misma la forma fue cosa de mirar en mis estantes, en particular el ejemplar de Nostálgicas Mansiones, de Teófilo Cid. A partir de entonces, con El Árbol Ediciones, (2002) no he podido parar de editar, no he podido clausurar ese boliche; no he querido ceder el breve espacio en que sí estoy.

\section{VII}

Pero cuando me identifico como autoeditora, no solo me aliento con los grandes que se han pagado su propia edición, como Juan Rulfo o Antonio Porchia; o los que verdaderamente se han autoeditado, como William Blake, Virginia Woolf, Margaret Randall. Pienso en mis colegas aquí mismo en Valparaíso, como Gladys González (Libros del cardo) o Priscilla Cajales (Hebra Editorial) quienes, impriman o no obra propia bajo sello propio, son ediautoras porque ejercen una personalidad a través de sus decisiones editoriales que no serían las mismas si no fueran sus propias jefas.

\section{VIII}

Cuando inicié con El Árbol Ediciones en Monterrey, México, cuya área metropolitana supera los cuatro millones de habitantes, no había más que dos o tres editoriales independientes, surgidas al alero de publicaciones periódicas. Esto puede deberse a la presencia de una casa editorial universitaria abierta a nuevos autores, coediciones, y muy prolífica, como es la Universidad Autónoma de Nuevo León, que junto a otras editoriales universitarias, conjugadas con el Fondo de Cultura Económica y el libro desgravado, han proporcionado cierto contrapeso a la dinámica del libro mercancía de las últimas décadas.

\section{IX}

Dos proyectos de El Árbol Ediciones enfrentaron problemas específicos del libro en México: la colección Signos del Umbral y La Ventana Ilustrada. El primero atacaba la desconexión entre los estados de México, que se relacionan a través del centro, publicando y difundiendo recíprocamente a un poeta de Nuevo León y otro michoacano. El segundo fue un proyecto desarrollado específicamente para fomento lector en cárceles, de la mano de una asociación de apoyo a presos de escasos recursos, con un número dirigido al encierro masculino y otro al de las mujeres. 


\section{La respuesta al modelo neoliberal}

\section{I}

Un amigo que vivió una temporada en Veracruz, México, me decía que le llamó la atención lo poco involucrada en política que era la gente. A mí me pasó a la inversa: cuando volví luego de un par de décadas en México, Chile me pareció un país ideológicamente inflado. Como si todo pasara por ahí, con una presión permanente de establecer una postura ideológica, que no se siente en un país donde las identidades locales son tan espesas, que llevan al lugar común de "México es muchos méxicos".

Aquí se habla de los "dos chiles", en referencia a la extrema desigualdad socioeconómica. Allá lo más parecido es la división entre el norte enblanquecido rico y el sur indígena empobrecido; pero no agota la gran diversidad, el pequeño cosmos de cada pueblo, íntimamente ligado a la diversidad de herencia indígena: se hablan más de 365 lenguas y dialectos. Y aunque actualmente los hablen solo el 6.6 porciento de la población, el dato engaña respecto a lo identitario, como lo explica la escritora Yásnaya Elena A. Gil: "Lo que ahora llamamos una mayoría mestiza es población que en realidad es indígena, pero que fue desindigenizada a través de arrebatarles la lengua." ${ }^{\text {i }}$

\section{II}

Si la escasa población indígena en Chile, en la que subsiste el apellido aún cuando no se hable la lengua, ha logrado hacerse escuchar en los últimos tiempos, ha sido por dos vías que se cruzan: la causa mapuche ligada a otras reivindicaciones, y la difusión de ciertas nociones entre la población ajena a su cultura. Es decir que el componente identitario y el componente ideológico se mezclan al hacer causa común con el ecofeminismo o con el movimiento estudiantil, por ejemplo.

\section{III}

La pulsión ideológica que acompaña a una resistencia política al acendrado modelo neoliberal chileno, creo yo, guarda relación con el surgimiento en Chile de editoriales independientes que buscan en el libro maneras de ocupar lo público. Con el declive de las editoriales nacionales y la polarización de las transnacionales de un lado y las independientes de otro, se diría que la globalización azuzó a una jauría editorial ciudadana, que conquistó ciertos espacios pero que aún no logra romper la brecha que la separa 
del ancho mundo o largo país de poetas que no distingue nombres de autores reconocidos, menos aún desconocidos, o editoriales.

\section{IV}

Algo similar sucedió en Argentina, como lo relata Gladys González (Libros del cardo) en entrevista: "En Argentina en los 90 hubo una explosión de editoriales por la crisis económica. Aparecieron las cartoneras, Cucurto, Cecilia Pavón, que tenían un espacio llamado "Belleza y felicidad”. Desde ahí irradiaban instancias de microeditoriales, fanzines, fotocopiados, distintos formatos. Yo ya tenía la intención de hacer una editorial pero no sabía cómo. Entonces las editoriales independientes eran de hombres. A partir del 2010 vino un boom de editoriales."

\section{¿Por qué editar?}

a)

Editar en estos tiempos supone mucho más que defender un objeto pasado de moda y su halo sagrado de baluarte del conocimiento y la cultura, contra la futilidad del espectáculo y las redes. Más que aferrarse a una posteridad, una permanencia, en medio de la avalancha de objetos desechables. Editar supone plantearse una idea de ciudadanía ante un Estado que se desmorona, y una búsqueda de nuevas comunidades. Implica un intento de articular el conocimiento, la estética, que circula disperso en un mercado de especulación, asediado por la inmediatez.

b)

Una comunidad de lectores es en sí una paradoja, si pensamos en la comunidad como un ente de convivencia oral, y en la lectura como una práctica ciudadana. Ese lector ideal, desconocido, particular, al que el libro ofrece su estandarización, su existencia en serie, no tiene rostro ni edad, ni siquiera tiene idioma, por cuanto un libro, una vez escrito, es susceptible de ser traducido.

c)

Con las redes hemos aprendido otras configuraciones de lo público, en que no es sinónimo de cantidad. Lo público también quiere decir camino de llegada a los contenidos. Un ejemplo de esto es el seguimiento que hacen los medios tradicionales de comunicación de las cuentas privadas de redes. Cada cuenta es como una revista en sí misma con contactos a cargo de secciones de humor, de cocina, de poesía, de 
política. Mundos semipúblicos que se entrelazan, semiverbales sono-pictográficos. El poeta Macedonio González desde hace años publica en su Facebook únicamente sus textos y no los publica en ningún otro lugar, con una veintena de sus 1719 amigos interactuando con ellos.

d)

Dice María Zambrano (1950) "Comunidad de escritor y público que, en contra de lo que primeramente se cree, no se forma después de que el público ha leído la obra publicada, sino antes, en el acto mismo de escribir el escritor su obra. Es entonces, al hacerse patente el secreto, cuando se crea esta comunidad del escritor con su público. El público existe antes de que la obra haya sido o no leída, existe desde el comienzo de la obra, coexiste con ella y con el escritor en cuanto a tal. Y sólo llegarán a tener público, en la realidad, aquellas obras que ya lo tuvieren desde un principio" (p. 43). De este modo editar vendría siendo realizar un público, concretar lectores, imprimir comunidad.

f)

Editar no es postular a un fondo para imprimir: es postular un espacio simbólico para ser habitado y hacer más habitable el mundo. Editar es evadir la inmediatez. Publicar es fugarse del círculo de amigos. Hay por tanto en ello un deseo de intervención pública, una aspiración ambiciosa: un espacio en el tiempo.

\section{g)}

En la edición hay también otra forma de ejercicio ciudadano: el oficio. La organización de ese espacio simbólico y sus convenciones. Un amor a la tipografía, una inclinación por los paralelepípedos. También hay una ilusión, vana, de procurarse un ingreso más estable que el de la escritura. El gran beneficio del libro, es que, si es bueno, dura más que los congelados. No hay merma.

h)

La apertura a los nuevos autores, la visibilidad de las autoras, el rescate de textos soslayados, la traducción y el vínculo con otras culturas, la inclusión de escrituras indígenas y migrantes son algunas de los esfuerzos de las ediciones independientes por llevar al espacio público los temas y las voces que no tienen acceso al círculo de producción del libro mercantilizado. 
Se dice que son las editoriales independientes las que sostienen la bibliodiversidad.

Creo que es esa misma responsabilidad la que convierte a la edición independiente en una forma de resistencia cívico-comunitaria. Pero la bibliodiversidad, que se supone custodiamos es solo la punta del iceberg de una diversidad sofocada por un modelo en erosión. En esa comunidad millonaria de lectoras y lectores que aprendieron la lengua del amo, hay mucha escritura por representar.

\section{Referencias bibliográficas}

Gil, Y. (2021). Frontera de la lengua / Entrevistada por Karla Sánchez. Letras Libres (26), 44-47. https://www.letraslibres.com/mexico/revista/entrevista-yasnaya-elena-gil-lalengua-tiene-una-carga-politica

Zambrano, M. (1950). Hacia un saber sobre el alma, Madrid, Alianza Editorial. 